\title{
Performance Analysis of Multi-hop and Cluster Based Routing Protocols for Mobile Heath Monitoring
}

\author{
MARYAM EL AZHARI ${ }^{1}$, AHMED TOUMANARI ${ }^{2}$ and RACHID LATIF ${ }^{3}$ \\ 1,2,3 ESSI, National School of Applied Sciences, Ibnou Zohr University, Agadir Morocco \\ E-mail: ${ }^{1}$ maryam.ensa@gmail.com, ${ }^{2}$ atoumanari@yahoo.fr, ${ }^{3}$ latif_rachid@yahoo.fr
}

\begin{abstract}
This paper aims to depict the performance of cluster based routing protocol (Leach protocol) and multipath routing protocol (Multipath Ring Routing protocol) for Wireless Body Sensor Networks where the mobility is a crucial constraint to handle. Previous works mainly dedicate the above mentioned protocols to Wireless Sensor Networks where the topology is static and support hundreds of sensors, however, their performance is expected to highly degrade when it comes to a mobile environment including a limited number of sensors. The results proved that multipath routing performs better than cluster based routing in both static and mobile topology.
\end{abstract}

Keywords: Wireless Body Sensor Networks, Routing Protocols, Cluster Based Protocol, Multipath Routing Protocol, Mobile Networks.

\section{INTRODUCTION}

Wireless Body Sensor Networks is a special kind of Sensor Networks characterized by a short range of transmission and a limited number of in-body (implanted or swallowed) sensors and on-body sensors capable of monitoring medical and non medical applications using multi-hop and single hop topologies. Several protocols have been proposed to handle data packets routing in Wireless Sensor Netwoks [1], however these protocols cannot be applied directly to Wireless Body Sensor Networks since additional constraints are added such as : Energy supply, medical sensors tends to have a small size compared to normal sensors dispersed in fields, which implicates smaller battery size, thus a certain amount of energy consumed within Sensor Networks might lead to a decrease of battery charge corresponding to " $x$ " units, these units might lead to a complete dysfunctioning of a medical sensor networks, for this, it is plausible to choose the most suitable routing protocol to medical application. Another important constraint is: Mobility: the body experiences high mobility, which takes a variety of models such as :SITTING, WALKING, LAYING-DOWN, RUNNING,etc.., medical sensors are supposed to have small power of transmission in order to minimize the tissue heating effect, thus a very mobile environment leads to a continuous disconnection of sensors increasing though its inefficiency in terms of throughput, end-to-end delay and energy consumption. Data priority is a factor that should be taken into consideration when implementing a routing protocol for WBSNs. EEG and ECG signals have to be delivered with high priority especially for persons with critical situation [2] . Most of routing protocols proposed for Wireless Sensor Networks are based on a static topology where path establishment is performed during the set up phase, however when it comes to a mobile environment, the set up phase might experience serious packet loss which degrades the throughput of the corresponding routing protocol. In our work, we aim to shed the light on the performance of following routing protocols: LEACH as a cluster based and MULTIPATH RING as multipath based routing protocol. The reason behind this analysis is first of all finding out which type of routing protocols is well suited for data delivery in Wireless Body Sensor Networks, and second of all preparing a solid ground to implement a new routing protocol responding to WBSNs constraints based on the results analysis. The paper is organized as follows: section 2 presents an overview of Wireless Body Sensor Networks 
applications, section 3 presents the functioning mechanism of LEACH and MULTIPATH RING routing protocols. An analysis of the performance is given in section 4; finally Section 5 concludes this paper.

\section{OVERVIEW OF WIRELESS BODY SENSOR NETWORKS APPLICATIONS}

A Wireless Body Sensor networks (WBSNs) consist of multiple sensors located on the human body and combined with a local end point, it could be a Personal digital assistant (PDA), a cell phone or any end point capable of collecting, processing data and send it back to the base station where suitable treatments are taken place (see . Medical sensors are used in many applications such as:

\subsection{Asthma}

A body sensor networks is a very useful solution for patients having asthmatic seizures, it is based on deploying a specific number of sensor nodes on the body which sense allergens in the environment such as dust mite allergen, pet dander, pollen, mold, etc and transfer the results back to the doctors [3].

\subsection{Cancer Detection}

A body sensor networks is a very useful solution for patients having asthmatic seizures, it is based on deploying a specific number of sensor nodes on the body which sense allergens in the environment such as dust mite allergen, pet dander, pollen, mold, etc and transfer the results back to the doctors [4].

\subsection{Glucose Level Monitoring}

Continuous glucose monitoring (CGM) systems use a tiny sensor inserted under the skin to check glucose levels in tissue fluid. The sensor stays in place for several days to a week and then must be replaced. A transmitter sends information about glucose levels via radio waves from the sensor to a pager like wireless monitor. The user must check blood samples with a glucose meter to program the devices. Because currently approved CGM devices are not as accurate and reliable as standard blood glucose meters, users should confirm glucose levels with a meter before making a change in treatment [5].

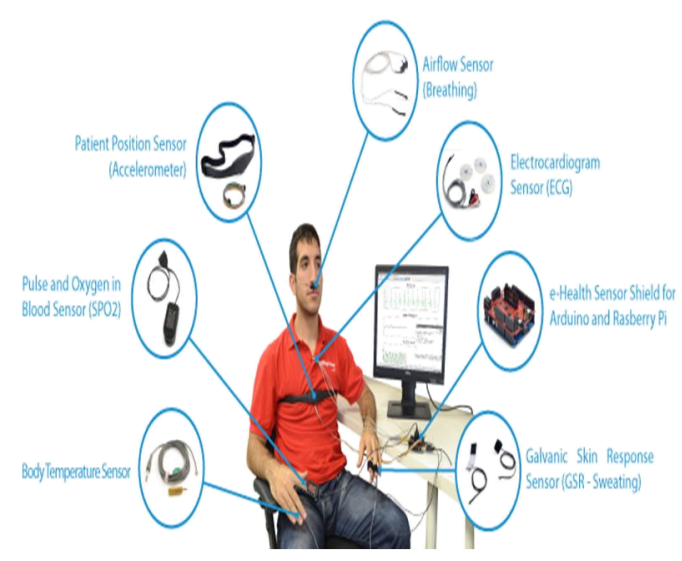

Fig. 1. Wireless Body Sensor Networks

\section{OVERVIEW OF LEACH AND MULTIPATH RING ROUTING PROTOCOLS}

\subsection{Leach Protocol}

Low-Energy Adaptive Clustering Hierarchy is a TDMA-based MAC protocol which is integrated with clustering and a simple routing protocol in wireless sensor networks (WSNs). The goal of LEACH is to lower the energy consumption required to create and maintain clusters in order to improve the life time of a wireless sensor network [6]. The main features of LEACH are:

\section{- Setting up Clusters}

- Rotating Cluster Heads role of the corresponding cluster

- Aggregation of data within a specific cluster and transmitting it back to the base station.

In Leach, sensors nodes are organized in clusters where a specific node is selected as a cluster head (see Figure 2 and Figure 3). Each node belonging to a cluster transmit its data to the corresponding cluster head data to that aggregates the data and transmit it back to the base station. LEACH is divided into rounds, and each round starts with a set-up phase followed by a steady phase. During the set up phase, each node sends information about its residual energy and its distance towards the sink node. Based on a central algorithm, the base station will then select and broadcast cluster head identifier 
to each sensor node. Using TDMA MAC protocol, CHs will then affect a time slot to each sensor member belonging the cluster and then broadcast the TDMA frame to all cluster members. The steady state start off after TDMA frame scheme transmission is finished and each sensor will send its data to the $\mathrm{CH}$ during its time slot. At the end of each frame when the cluster head has received data from all associated sensor nodes, it aggregates the data and sends it to the base station. The cluster heads send this data using a fixed spreading code with CSMA.

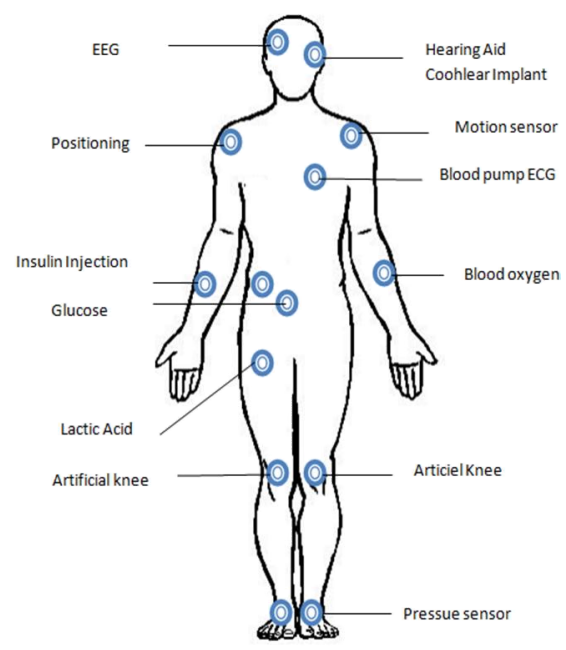

Fig. 2. Body Sensor Distribution

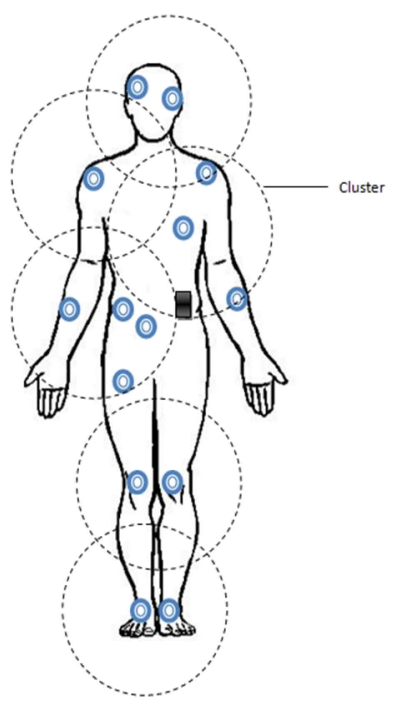

Fig. 3. Leach protocol

\section{MULTIPATH RING ROUTING PROTOCOL}

Multipath ring routing protocol consist of organizing the network into several levels based on hop count from the node to the sink so that each node will be part of a to a specific ring level [7]. Multipath ring routing protocol start off by establishing minimum paths from each sensor to the sink. In this phase, the sink node broadcasts a packet with its ring level 0 . The nodes which received the packet will increase their ring number and rebroadcast the packet to their neighbors. In the end, all the nodes in WSN are separated into several levels (see Figure 2 and Figure 4).

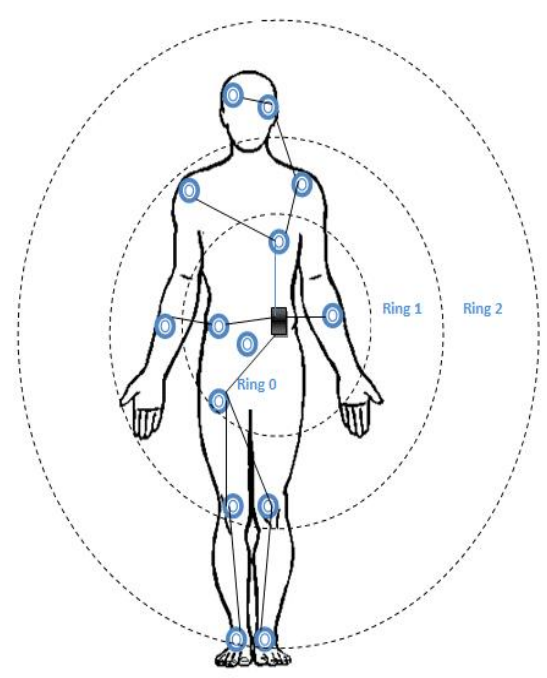

Fig. 4. Multipath ring routing protocol

\section{SIMULATION RESULTS}

In order to highlight the performance of LEACH and Multipath ring routing protocols, we run our simulation in Castalia 3.2 designed for Wireless Sensor Networks (WSN), Body Area Networks (BAN), and generally networks of low-power embedded devices [8-10]. We proceeded to analyze the performance of the above mentioned protocols in terms of latency, energy consumption and packet loss rate. The following parameters have been considered: Receiver (Rx) sensitivity equals to -87 $\mathrm{dBm}$, Transmitting power equals to $-25 \mathrm{dBm}$ (the reason for choosing $-25 \mathrm{dBm}$ as a transmitting power is to minimize the heating effect caused by using a high value of power transmission) ,12 
M. E. Azhari et. al / International Journal of Computer Networks and Communications Security, 2 (11), November 2014

sensor nodes distributed over the body with predefined locations including the PDA, and rate takes the following values respectively : $14,18,22,26,28$, and 30 . The simulation is run within static and mobile environment, for this, we choose Radom way mobility model to emulate the unpredictable movement of the body since it presents lowest latency and best packet delivery ratio compared with other mobility models[11].

\subsection{Leach protocol}

\subsubsection{Application Latency with Random Way Point Mobility Model}

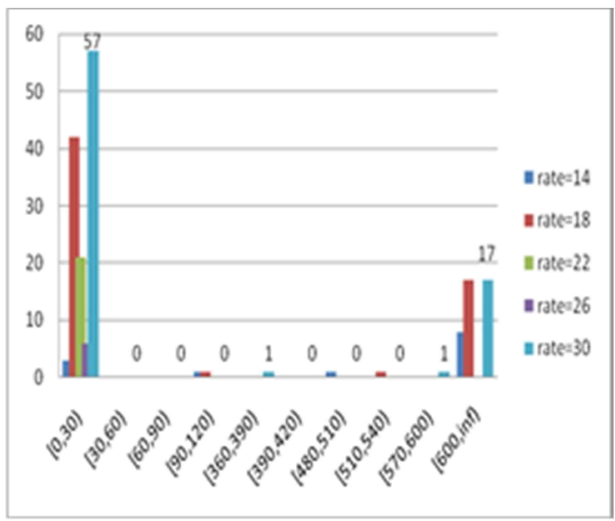

Fig. 5. Application Latency with RWPMM

Figure 5 shows application latency of leach routing protocol in a mobile environment. Most of the packets are received with latency inferior to 30 $\mathrm{ms}$ in respect to the rate with a maximum value equals to 57 packets versus 17 packets received above $600 \mathrm{~ms}$ in case of rate equals to 30. The results prove the absence of correlation between (rate, application latency) pair.

The explanation of this behavior is that the number of sensors within a cluster in a very mobile environment is variable, so during cluster construction, a cluster may contain an important number of sensors as it may include only few (if not any) sensors, so when applying TDMA MAC protocol during the set up phase, clusters with small size will experience a minimum latency compared with clusters with bigger size, which explains the results depicted in the graph above.

\subsubsection{Application Latency without model}

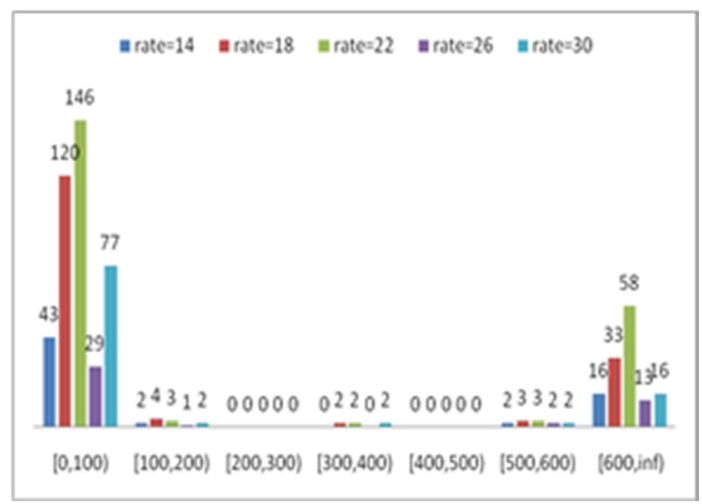

Fig. 6. Application latency without mobility model.

The Figure 6 depicts the performance of LEACH protocol in terms if latency in a static topology; it is observed that an important number of packets (max. 146) are received below $100 \mathrm{~ms}$ regardless of what value we have set for rate. A great amount is also received above $600 \mathrm{~ms}(\max .58)$, without neglecting the small portion at $[100,200)$, $[300,400)$ and $[500,600)$ intervals. An explication of this behavior is that after the creation of clusters, the corresponding members tend to maintain the connection to their cluster head, thus when sending data during the time slot granted by the cluster head, the packet has to wait (Number of Cluster Members - node ranking)* Timeslot before getting transmitted to the base station, thus, when the node ranking is high, the packet experiences an important latency compared with packets with low ranking i.e when the size of a cluster gets bigger, the average latency experienced by this cluster becomes bigger too compared with another cluster of smaller size which explain the diversity of packet reception latency.

\subsubsection{Energy consumption}

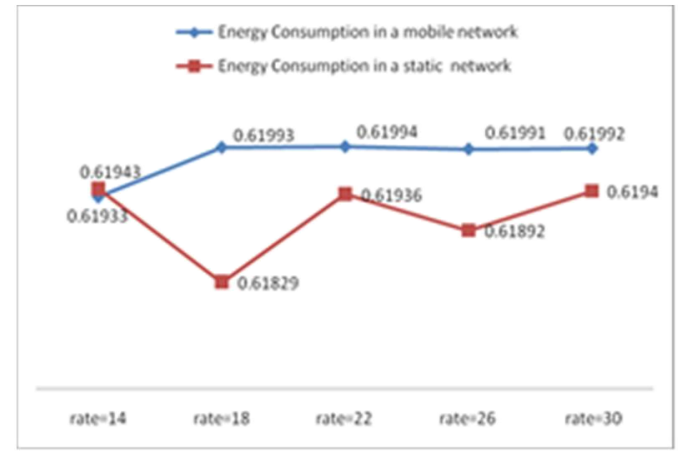

Fig. 7. Energy Consumption of Leach protocol 
As observed in Figure 7, the energy consumption is completely independent of rate. the average energy consumed for both mobile and static environment depends mainly on the amount of time the radio is on for both receiving and transmitting the packets, a small variation of the consumed energy can be observed for instance : $0.61933 \mathrm{mw}$ is the value consumed within a mobile environment when the rate equals to 14 versus 0.61993 for rate equals to 18 , this small variation is due to the energy consumed by CPU (central processing unit) to perform data aggregation and it depends also on the number of data to be aggregated.

\subsubsection{Packet Reception Ratio}

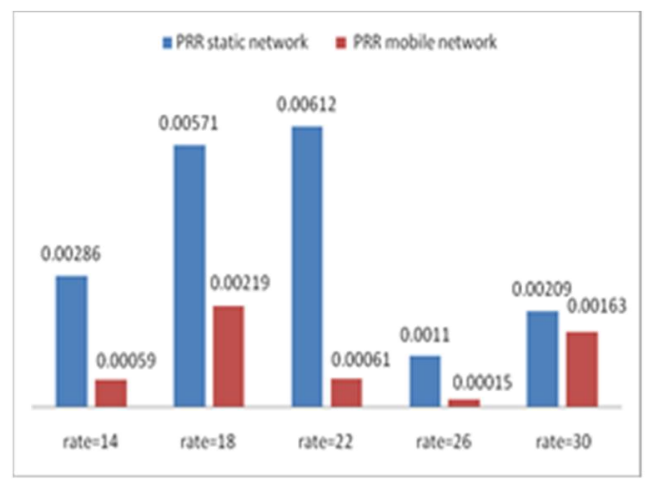

Fig. 8. Leach protocol Packet Reception Ratio

The packet reception ratio within a mobile environment is very low compared with a static environment, The packet reception ratio indicates the ratio of packets received out of all sent packets, a value converging to 1 is a good indicator compared with a value converging to 0 or above 1 , which represents either high packet loss or over reception of packets. Figure 9 highlights the absence of correlation between rate and PRR (Packet Reception Ratio), moreover PRR in both topologies is weak and shows a worse performance of LEACH protocol based on the simulation parameters depicted above, this high packet loss is caused by disconnectivity between sensor node and its destination, in case of static topology, nodes need more energy power to communicate control or data packets to either $\mathrm{CHs}$ or PDA, however higher transmission power leads to higher SAR (Signal Absorption Rate) which is considered hazardous for human heath, the mobility of sensors enhances sensors disconnectivity in a way that a mobile sensor might be out of destination communication range for a great amount of time which explains the results given in Figure 8.

\subsection{Multipath Ring Routing protocol}

\subsubsection{Application Latency with Random Way Point Mobility Model}

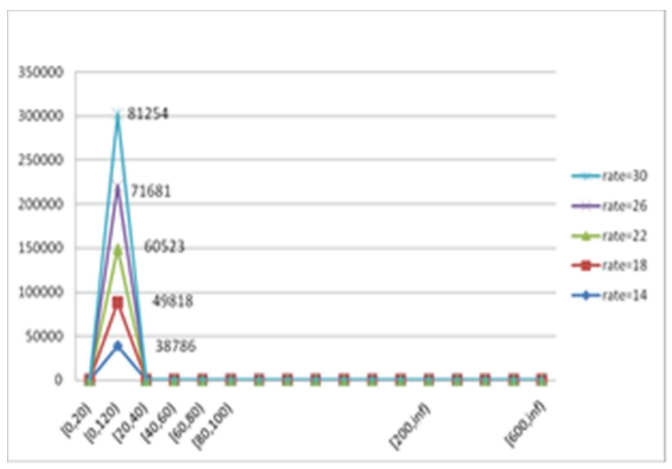

Fig. 9. Application Latency with RWPMM

\subsubsection{Application Latency without model}

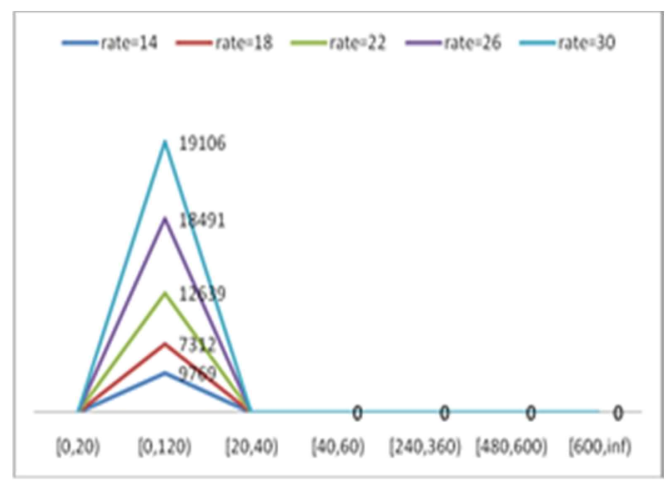

Fig. 10. Application Latency without mobility model

As it is shown in Figure 9 and Figure 10,as rate increases from 14 to 30,an important amount of packets (81254 in case of static network vs 19106 in mobile network) are received in time interval belonging to $[0,120)$,which is a good indicator especially when it comes to sensitive data requiring a minimum end to end delay, the advantage of multipath protocol is that packets are delivered via the shortest path to the PDA minimizing thus the delivery latency, in a mobile environment, the nodes belonging to such a path tends to be disconnected and an important number of nodes lost in a process this explains the great amount of packets received in static topology compared with a mobile one. 


\subsubsection{Energy consumption}

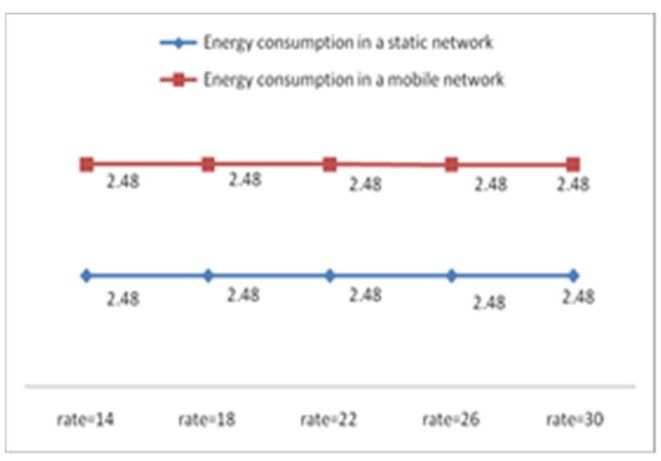

Fig. 11. Energy Consumption of Multipath Ring routing protocol

As shown in Figure 11, the average energy consumed is very high and equals to $2.48 \mathrm{~mW}$ but still the same within a mobile and a static environment. As mentioned before, the amount of energy consumed is independent from the amount of packet transmitted, the radio unit of a sensor consumes a much more energy than other sensor components (processing unit, sensing unit),it may have four states: transmitting state (TX),receiving state (RX), idle state (IDLE) and sleep state, the more a sensor is in TX/RX state the more it consumes energy, in our case, the sensor node is always maintaining a RX state if not transmitting in order to process and relay packets coming from upper layers (reprogramming packets ) and lower layers (data packets) which explains the constant value of energy consumption in respect to the rate.

\subsubsection{Packet Reception Ratio}

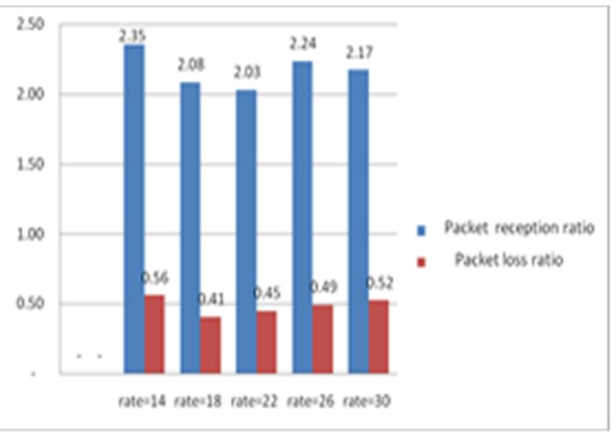

Fig. 12. Packet Reception Ratio of Multipath Ring protocol in a mobile environment

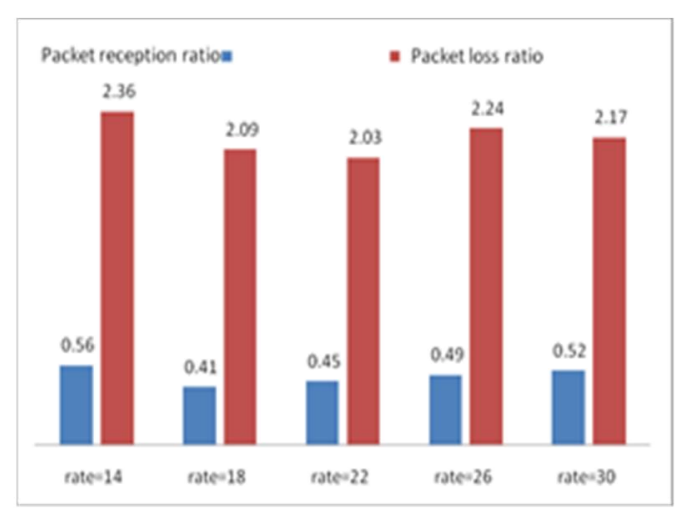

Fig. 13. Packet Reception Ratio of Multipath Ring protocol in a static environment

As observed in Figure 13, the packet reception rate varies in respect to the rate and reaches a maximum value equals to $2.35($ rate $=14)$ and a minimum value equals to 2.03 (rate $=22$ ), the PRR is higher than 1, which means that nodes are receiving packets with different level identification that are belonging to either upper layer leading to deprecation of this one or lower layer making the nodes broadcasting it to upper layer nodes towards the sink. However when it comes to a very mobile environment (Figure 14), the number of packet lost increases dramatically to reach a maximum value equals to 2.36 versus 0.56 for packet received successfully. An explication of this behavior is that within a mobile environement, the nodes are disconnected most of the time leading to a huge amount of packets lost in a process, also when sending a packet, the receiving nodes might be transmitting as well after the channel has been declared clear for transmission, thus packets experience high loss ratio due to interference but also due to the non reception state of radio nodes.

In order to perform a comparison between leach and multipath ring routing protocols, we proceeded with defining and calculating LEP (Latency, Energy and Packet loss rate product) and then choosing the routing protocol that presents the minimum value of LEP, the LEP formula is represented as follows:

$$
L E P=\sum_{i=1}^{n}\left(a_{i} \sum_{k=1}^{m} A_{k}+P L R_{i} \cdot E_{i}\right) / \mathrm{n}
$$


Where A represents the latency interval where packets are received is the number of intervals,, ai, bi $\epsilon\{0,1\}$, PLR : Packet Loss Rate, E: energy consumption, $\mathrm{n}$ :number of rate iterations . in our study, we were limited to $[0,120)$ and $[600, \infty)$ intervals since most of packets are received within these intervals in both leach and multipath ring protocols, The results proved that multipath ring routing protocol shows a better performance compared with leach protocol since it presents a minimum LEP equals approximately to 1.40 in a static environment versus 2.20 in a mobile environment (see Figure 14).

Where A represents the latency interval where packets are received is the number of intervals, ai $\epsilon$ $\{0,1\}$, PLR : Packet Loss Rate, E : Energy consumption:

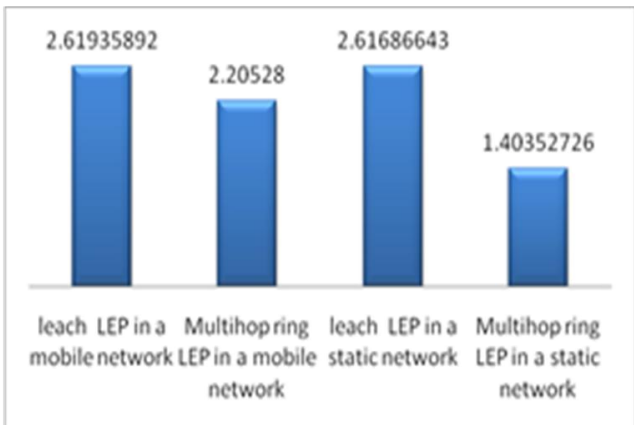

Fig. 14. LEP value for LEACH and MULTIPATH RING routing protocols

\section{CONCLUSION}

WBSNs provide promising applications in medical monitoring systems to measure specified physiological data but also non medical application and provide location-based information .In this paper, we presented a comparative study of cluster based and multihop based routing protocols in terms of energy consumption, end to end delay and packet reception ratio. The results demonstrated the high efficiency of multipath routing protocol compared to cluster based protocol in both static and mobile environment. As a future work, we will be focusing on creating a new routing protocol dedicated to a mobile topology based on multihop routing, to this end, special nodes called relay nodes are going to be deployed to relay packets to the PDA, the optimal number of relays has be defined in a way that the network cost and the energy consumption are reduced to the minimum guaranteeing thus a prolonged network lifetime.

\section{REFERENCES}

[1] S. Kumar Singh, M.P Singh and D K Singh, "Routing Protocols in Wireless Sensor Networks - A Survey ", International Journal of Computer Science \& Engineering Survey (IJCSES) Vol.1, No.2, November 2010 DOI: 10.5121/ijcses.2010.1206 63.

[2] Chi, Y.M. ,"Wireless Non-contact EEG/ECG Electrodes for Body Sensor Networks», Body Sensor Networks (BSN), 2010 International Conference on Date 7-9 June 2010.

[3] Pervez Khan, Md.Asdaque Hussain, Kyung Sup Kwak,"Medical Applications of Wireless Body Area Networks»), International Journal of Digital Content Technology and its Applications Volume 3, Number 3, September 2009.

[4] John McDevitt, Shannon E. Weigum, Pierre N. Floriano,Nicolaos Christodoulides, Spencer W. Redding, Chih-Ko Yeh,Stan McGuffey, Nadarajah Vigneswaran, Martin H. Thornhill, and Michelle D. Williams, "A new bionanochip sensor aids oral cancer detection ",SPIE, 10.1117/2.1201102.003547.

[5] Dr .S. Padmapriya, Abhishek Chowdary and V.S.Dinesh ,"Wireless sensor networks to monitor Glucose level in blood», International Journal of Advancements in Research \& Technology, Volume 2, Issue4, April-2013.

[6] Xiaoyan Cui, "Research and Improvement of LEACH Protocol in Wireless Sensor Networks",IEEE $2007 \quad$ International Symposium on Microwave, Antenna, Propagation, and EMC Technologies For Wireless Communications.

[7] Guimin Huang,Wujin Tao, Pingshan Liu, Siyun Liu ,"Multipath ring Routing in Wireless Sensor Networks», Proceedings of the 2nd International Symposium on Computer, Communication, Control and Automation (ISCCCA-13)

[8] Castalia website:http://castalia. npc. nicta. com. au/

[9] K. Kwak, S. Ullah, and N. Ullah, "An Overview of IEEE 802. 15. 6 Standard ",UWBITRC Center, Inha University 253 Yonghyundong, Nam-gu, Incheon (402-751), South Korea,20 Feb 2011.

[10] Kyeong Hur, Won-Sung Sohn, Jae-Kyung Kim and YangSun Lee, "IEEE 802. 15. 6 WBAN Beaconing for Wireless USB Protocol Adaptation", International Journal of Software Engineering and Its Applications, Vol. 7, No. 4, July, 2013. 
M. E. Azhari et. al / International Journal of Computer Networks and Communications Security, 2 (11), November 2014

[11]C. Tracy, B. Jeff, D.Vanessa, "A Survey of Mobility Models for Ad hoc Network Research. Special Issue on Mobile Ad hoc Networking: Research, Trends and Applications," Journal of Wireless Communications and Mobile Computing, 2002, pp. 483-502. 